\title{
Finite size scaling for 3 and 4-flavor QCD with finite chemical potential
}

\section{Shinji Takeda ${ }^{* a, b}{ }^{\dagger}$ Xiao-Yong $\operatorname{Jin}^{b}$, Yoshinobu Kuramashi ${ }^{b, c, d}$, Yoshifumi Nakamura ${ }^{b}$, and Akira Ukawa ${ }^{d}$}

${ }^{a}$ Institute of Physics, Kanazawa University, Kanazawa 920-1192, Japan

${ }^{b}$ RIKEN Advanced Institute for Computational Science, Kobe, Hyogo 650-0047, Japan

${ }^{c}$ Graduate School of Pure and Applied Sciences, University of Tsukuba, Tsukuba, Ibaraki 305-8571, Japan

${ }^{d}$ Center for Computational Sciences, University of Tsukuba, Tsukuba, Ibaraki 305-8577, Japan

\begin{abstract}
We present a finite size scaling study for 3 and 4-flavor QCD with nonzero quark chemical potential by the grand canonical approach. We employ the Wilson-clover fermions and adopt the phase reweighting method where the phase factor is computed exactly. In 4-flavor study, we show the finite size scaling study for the moments of various physical quantities, which demonstrates a typical behavior of the 1 st order phase transition as well as the crossover or weak 1 st order phase transition. For 3-flavor theory, we study the critical end point in the bare parameter space spanned by the hopping parameter, the gauge coupling and the chemical potential.
\end{abstract}

31st International Symposium on Lattice Field Theory LATTICE 2013

July 29 - August 3, 2013

Mainz, Germany

\footnotetext{
* Speaker.

†E-mail: takeda@hep.s.kanazawa-u.ac.jp
} 


\section{Introduction}

The 4-flavor theory is known to be a good testing ground to study QCD phase diagram with finite density [ [U, 口, 四, 团] since the phase structure of the 4-flavor theory is expected to be very similar to that of the 3-flavor theory. For example, Fig. (left) is an expected phase diagram for the low flavor theory in the massless limit where a 1st oder phase transition line is connecting the temperature and the chemical potential axis. By increasing the quark mass, the 1st order phase transition at zero density becomes weaker while the transition at zero temperature is expected to remain 1st order, therefore the 1st order transition line turns into a crossover and then a critical end point appears at some chemical potential as shown in the right panel of Fig.⿴囗十. In 4-flavor case, however, it is empirically known that the 1st order phase transition at zero density persists even in relatively heavy mass region [[]]. Therefore the critical end point is likely located near the temperature axis with small chemical potential compared with that of the low flavor case. Then the analysis of the critical end point should be easier with less sign problem. Furthermore, since the parameter space we are interested in is in heavy mass region, the simulation cost should be reasonable.

In this report, the moment analysis for 4-flavor theory is given and we show some typical examples for 1st order phase transition and crossover/weak 1st order phase transition. The details of the analysis can be found in Ref.[6]. The Lee-Yang zero analysis by using the same data set is reported in $[\mathbb{⿴ 囗 十 ]}$. We also present a preliminary study of the 3-flavor theory especially focusing on the determination of the critical end point.
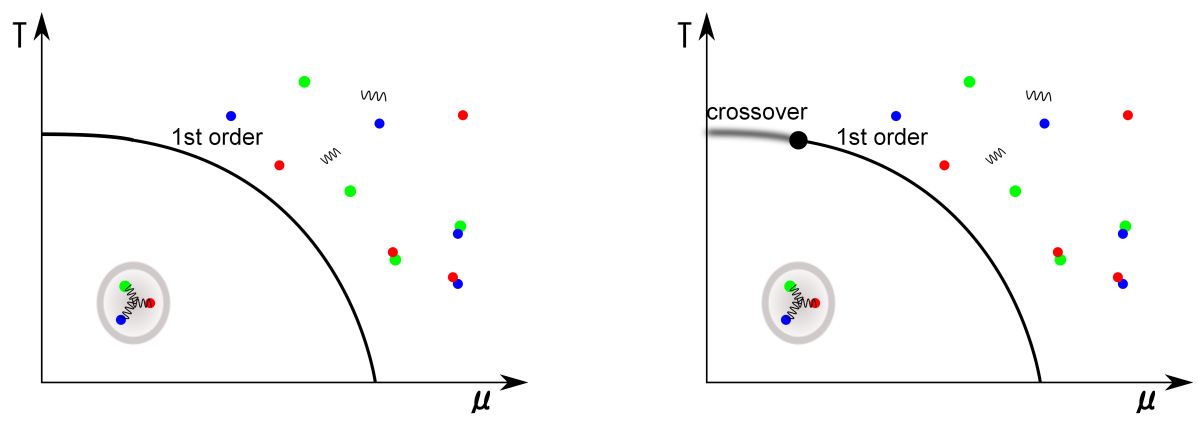

Figure 1: Expected phase diagram at the massless limit (left) and relatively heavy mass (right).

\section{Simulation setup}

We adopt the grand canonical approach and use the phase reweighing method to avoid the complex action problem. The gauge configurations are generated by following the phase-quenched QCD partition function,

$$
\mathscr{Z}_{\|}(\mu)=\int[d U] e^{-S_{\mathrm{G}}}|\operatorname{det} D(\mu)|^{N_{\mathrm{f}}},
$$

where the quark mass and chemical potential are set to be identical for all flavors and $N_{\mathrm{f}}$ is the number of flavor. The lattice action is a combination of the Wilson-clover fermion action and the 
Iwasaki gauge action. For both $N_{\mathrm{f}}=3$ and 4 , we use the clover coefficient $c_{\mathrm{sw}}$ which is nonperturbatively tuned for $N_{\mathrm{f}}=3$.

The physical quantities are estimated by the reweighting formula,

$$
\langle\mathscr{O}\rangle=\frac{\left\langle\mathscr{O} e^{i N_{\mathrm{f}} \theta}\right\rangle_{\|}}{\left\langle e^{i N_{\mathrm{f}} \theta}\right\rangle_{\|}}
$$

The complex phase is computed by using the reduction technique proposed in Ref.[[]] with additional improvements and obtain the phase exactly. GPGPU played crucial role to accumulate large statistics in this hot spot of the calculation.

\section{Simulation results for $N_{\mathrm{f}}=4$}

In the $N_{\mathrm{f}}=4$ study, the temporal lattice size is fixed at $N_{\mathrm{T}}=4$ and two sets (called light mass and heavy mass) of parameters are used as follows:

\begin{tabular}{c|c|c|c|c|c|c} 
& $\beta$ & $\kappa$ & $a \mu$ & $N_{\mathrm{L}}^{3}$ & $m_{\pi} / m_{\rho}$ & $T / m_{\rho}$ \\
\hline light mass & 1.58 & 0.1385 & $6^{3}-10^{3}$ & $0.02-0.30$ & 0.822 & 0.154 \\
heavy mass & 1.60 & 0.1371 & $6^{3}-8^{3}$ & $0.10-0.35$ & 0.839 & 0.150
\end{tabular}

\subsection{Phase reweighing factor}

Fig. $[$ is the phase-reweighting factor as a function of $a \mu$ for the light mass and heavy mass cases. This quantity is useful to see a situation of the sign problem. For large $\mu$ and large volume, the value tends to be close to zero then the sign problem is getting harder as expected. Nevertheless, it stays away from zero in some region, thus phase reweighing is still useful there. There are a dip and hump for both masses and they are considered as an effect of transition of QCD and phase quenched QCD respectively and the reasoning is given in [6].
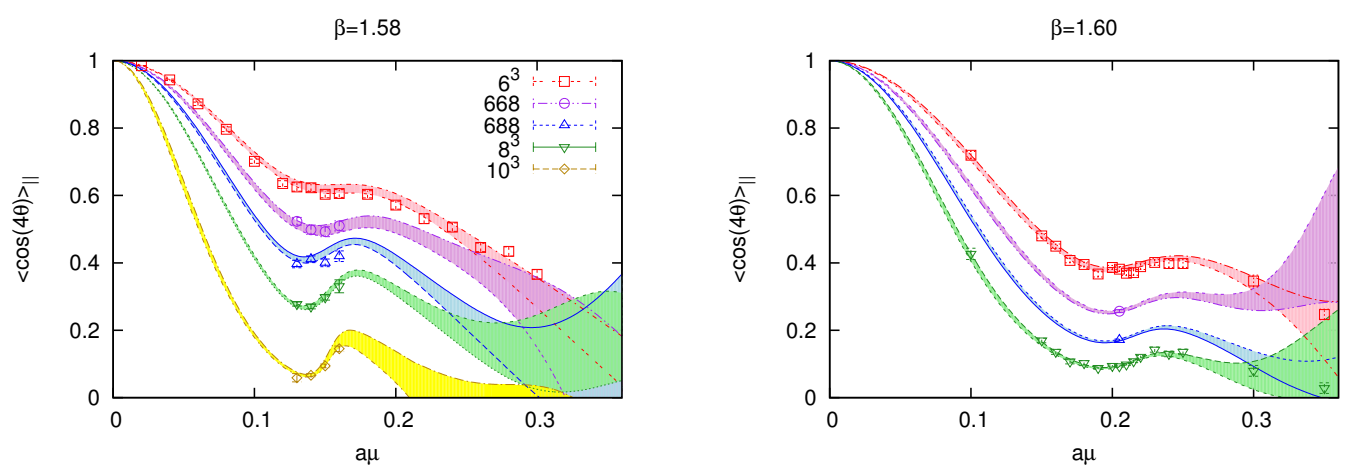

Figure 2: The phase reweighting factor as a function of $a \mu$ for light mass (left) and heavy mass (right) case. The band is $\mu$-reweighted result. 


\subsection{Susceptibility}

Fig.[3] is the volume scaling of susceptibility peak. For lighter mass, we can clearly see a linear volume dependence and this suggests a 1st order phase transition and the crossover is strongly excluded. For heavy mass, all fitting forms are consistent with the data and it is very hard to distinguish between the cross over and very weak 1st order phase transition. Thus we cannot draw a clear conclusion with our current volume.
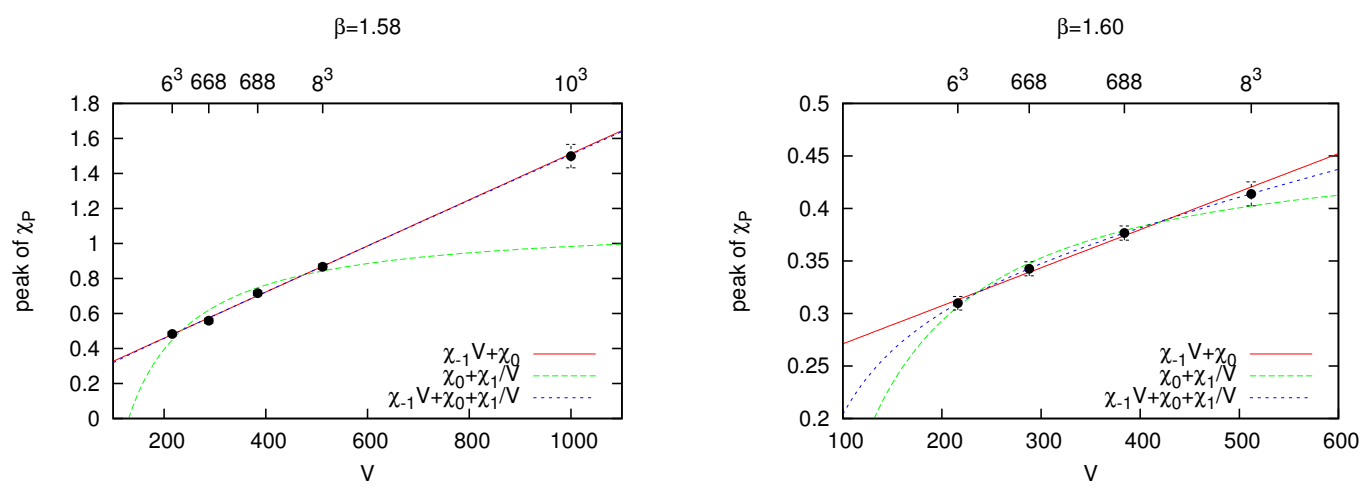

Figure 3: The volume scaling of the susceptibility peak for plaquette value. The left (right) panel is for light (heavy) mass case.

\subsection{CLB cumulant}

To consolidate a conclusion about the order of the transition, we show another quantity, ChallaLandau-Binder cumulant [Q, 四], defined by

$$
U_{X}=1-\frac{1}{3} \frac{\left\langle X^{4}\right\rangle}{\left\langle X^{2}\right\rangle^{2}}
$$

for a physical quantity $X$. This also has a criteria for the strength of transition like here.

$$
\lim _{V \rightarrow \infty} U_{\min }=\left\{\begin{array}{cl}
2 / 3 & : \text { crossover } \\
\text { others } & : 1 \text { st order phase transition }
\end{array}\right.
$$

Fig. आ is a volume scaling for the minimum of CLB cumulant associated with the plaquette average. The light mass case shows a clear $1 / V$ scaling and the minimum value in the thermodynamic limit is significantly different from $2 / 3$, therefore it is consistent with the previous susceptibility analysis. For heavy mass case, it is again difficult to draw a clear distinction between the crossover and 1st order phase transition with our volumes.

\subsection{Global picture of phase diagram}

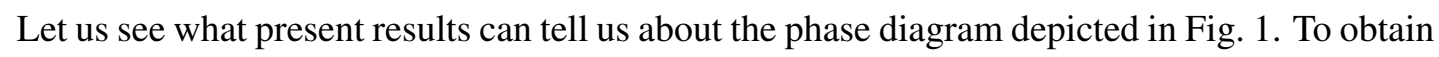
global picture of the phase diagram, we made additional simulations at $a \mu=0$ with $\kappa=0.1380$ and 0.1371 , while varying $\beta$ to search for transition points. And then it turns out that

$$
(\beta, \kappa, a \mu)= \begin{cases}(1.59997(11), 0.1380,0) & \text { strong } 1 \text { st order phase transition } \\ (1.61848(12), 0.1371,0) & \text { very weak 1st order/cross over. }\end{cases}
$$



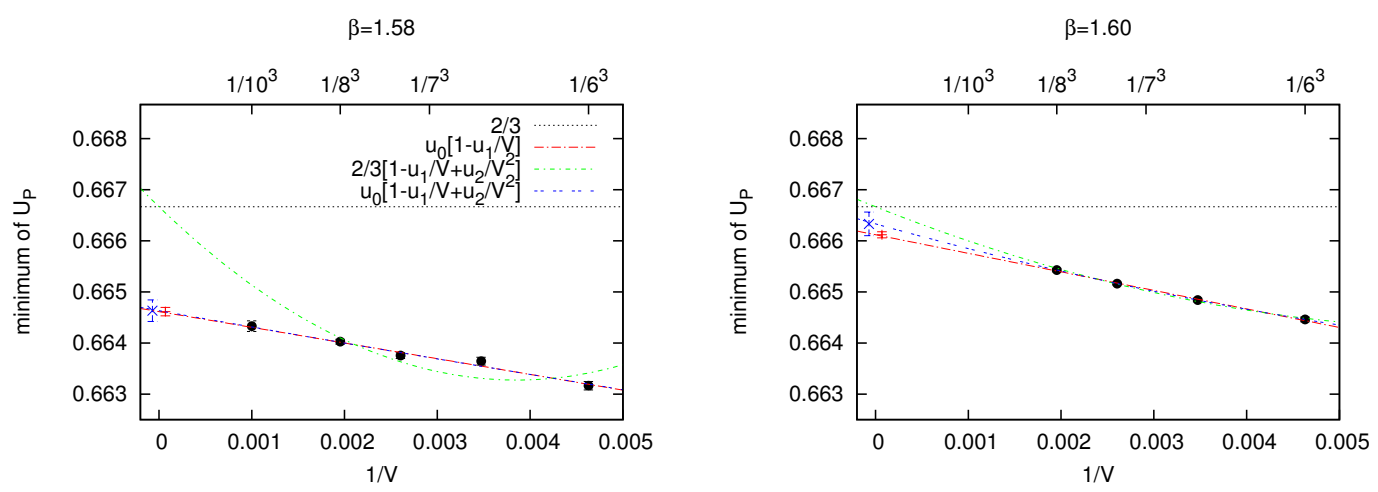

Figure 4: The volume scaling of the minimum of CLB cumulant for plaquette value. The left (right) panel is for light (heavy) mass case.

In addition to the above points, the transition points at finite density

$$
(\beta, \kappa, a \mu)= \begin{cases}(1.58,0.1385,0.1459(20)) & \text { strong 1st order phase transition } \\ (1.60,0.1371,0.2053(21)) & \text { very weak 1st order/cross over }\end{cases}
$$

are plotted in the phase diagram of Fig. [. At zero density, along the transition line, continuity suggests that a strong 1st order transition at stronger couplings (lower-right corner of the figure) weakens toward weaker couplings (upper-left corner). To understand the phase diagram related to finite densities in terms of physical observables, we calculate $m_{\pi} / m_{\rho}$, and linearly interpolate lines of constant $m_{\pi} / m_{\rho}$. We pick specifically $m_{\pi} / m_{\rho}=0.822$ and 0.839 evaluated at the transition points at finite densities, and show these two lines in the figure as green and magenta lines, respectively. A crossing point of such a constant $m_{\pi} / m_{\rho}$ line and the zero density transition line (black) gives us an estimate of the location of the zero density thermal transition at the value of $m_{\pi} / m_{\rho}$, which in turn tells us the relative strength of the transition there.

In Fig. 1, at the intersection of the black and the green lines, which has a stronger coupling than our simulated point (open square) at zero density, we expect a strong 1st order transition, which continues to $a \mu=0.1459$ (20) (filled square), for light mass system with $m_{\pi} / m_{\rho}=0.822(2)$ along the green line. Therefore, the phase diagram for $m_{\pi} / m_{\rho}=0.822(2)$ looks like the left panel of Fig. 四.

On the other hand, for the system with heavy mass $m_{\pi} / m_{\rho}=0.839(2)$, we have argued that it has either a weak 1st order transition or a quick cross-over at $a \mu=0.2053$ (21) (filled diamond). Extending toward the zero density (black) line, the system appears at much weaker coupling than our simulated weak transition point (open diamond), and is likely to have an even weaker transition or a quick cross-over. The phase diagram for $m_{\pi} / m_{\rho}=0.839(2)$ is, therefore, possibly consistent

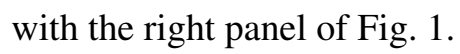

\section{Simulation results for $N_{\mathrm{f}}=3$}

Our purpose for $N_{\mathrm{f}}=3$ study is slightly different from the 4-flavor case and it is to identify the location of the critical end point at the finite density. A strategy to search for the critical end 


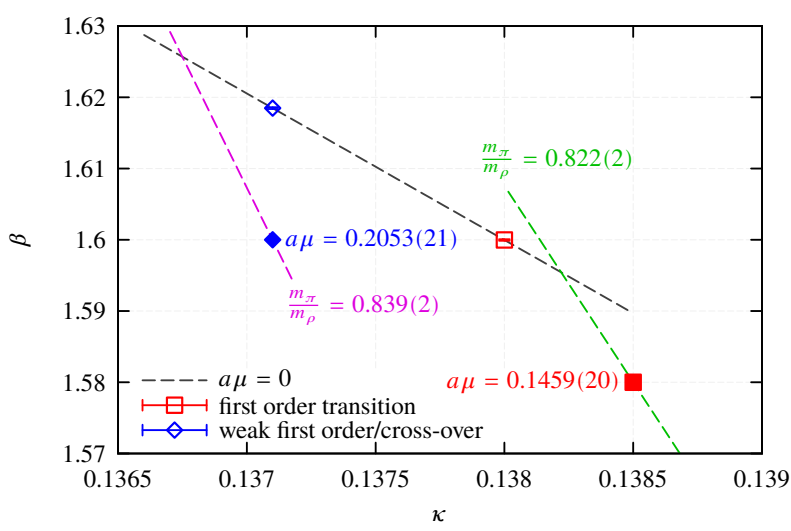

Figure 5: The phase diagram in the bare parameter space. The black line is the transition line at zero density.

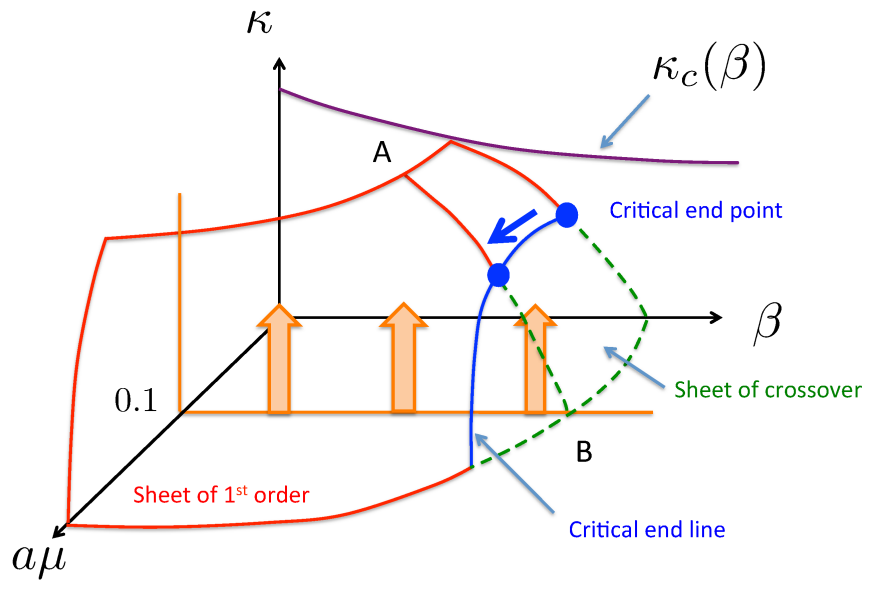

Figure 6: A strategy to search for the critical end point at finite density.

point is sketched in Fig.6. First, we identify the location of the critical end point at zero density whose details are given in a contribution of proceedings of Lattice 2013 [ए2]. Then by switching on the chemical potential, we trace the critical end point which draws a line in the 3-dimensional bare parameter space, we call it critical end line. At fix $a \mu=0.1$, we basically follow the same procedure at zero density, namely in the $\kappa-\beta$ plane, we identify the transition line (A-B line in Fig.(6) by varying $\kappa$ for each $\beta$.

The 3-flavor simulation was done on the lattice with $N_{\mathrm{T}}=6, N_{\mathrm{L}}=8,10,12$. The left panel of Fig. $\square$ is the volume scaling of the susceptibility peak for the gauge action density along the transition line. This shows that along the transition line from the strong coupling to weak coupling the 1st order phase transition turns into crossover and this is consistent with the scenario in Fig.6 (from A to B). Therefore there should be a critical end point in between $\beta=1.70$ and 1.77. In order 
to precisely identify the location of the critical end point, we adopt the kurtosis (Binder cumulant) intersection method [प]]. This method tells that if individual lines for each volume cross each other then the position where the crossing occurs is considered to be the critical end point. In the right panel of Fig.W, the lines for $N_{\mathrm{L}}=8$ and 10 have a crossing point while 10 and 12 do not have a clear one. It seems that we need more points of $\beta$ and larger volumes to identify the critical end point.
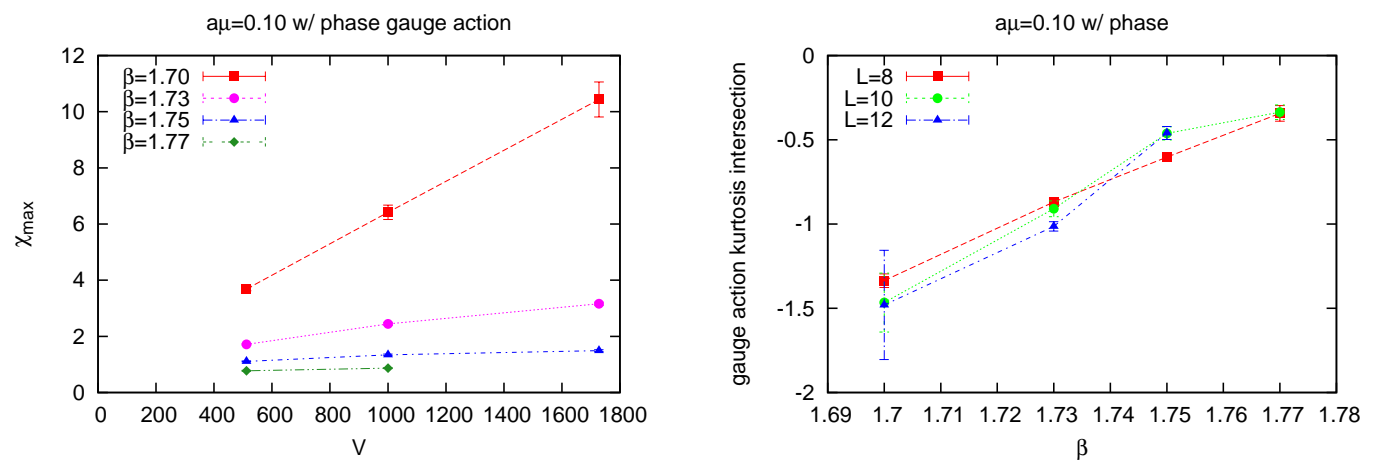

Figure 7: The volume scaling of the susceptibility peak for gauge action density (left). The intersection of kurtosis for gauge action density (right).

This work is supported in part by the Grants-in-Aid for Scientific Research from the Ministry of Education, Culture, Sports, Science and Technology (Nos. 23105707, 23740177, 22244018, 20105002). The numerical calculations were done on T2K-Tsukuba and HA-PACS cluster system at University of Tsukuba.

\section{References}

[1] Z. Fodor and S. D. Katz, Phys. Lett. B 534, 87 (2002) [hep-lat/0104001].

[2] M. D’Elia and M. -P. Lombardo, Phys. Rev. D 67, 014505 (2003) [hep-lat/0209146].

[3] S. Kratochvila and P. de Forcrand, PoS LAT 2005, 167 (2006) [hep-lat/0509143].

[4] A. Li, A. Alexandru, K. -F. Liu and X. Meng, Phys. Rev. D 82, 054502 (2010) [arXiv:1005.4158 [hep-lat]].

[5] M. Fukugita and A. Ukawa, Phys. Rev. Lett. 57, 503 (1986).

[6] X. -Y. Jin, Y. Kuramashi, Y. Nakamura, S. Takeda and A. Ukawa, arXiv:1307.7205 [hep-lat].

[7] X. -Y. Jin, Y. Kuramashi, Y. Nakamura, S. Takeda and A. Ukawa, arXiv:1311.2756 [hep-lat].

[8] J. Danzer and C. Gattringer, Phys. Rev. D 78, 114506 (2008) [arXiv:0809.2736 [hep-lat]].

[9] M. Fukugita, M. Okawa and A. Ukawa, Nucl. Phys. B 337, 181 (1990).

[10] M. S. S. Challa, D. P. Landau and K. Binder, Phys. Rev. B 34, 1841 (1986).

[11] F. Karsch, E. Laermann and C. Schmidt, Phys. Lett. B 520, 41 (2001) [hep-lat/0107020].

[12] X-Y. Jin, Y. Kuramashi, Y. Nakamura, S. Takeda and A. Ukawa PoS(Lattice 2013)138 